\title{
Umbilical cord blood plasma-derived exosomes as a novel therapy to reverse liver fibrosis
}

\author{
Yu-Jen Huang ${ }^{1} \mathbb{D}^{0}$, Jerry $\mathrm{Cao}^{2}$, Chih-Yuan Lee ${ }^{1}$ and Yao-Ming Wu ${ }^{1,3^{*}}$
}

\begin{abstract}
Background: Cirrhosis is a chronic liver disease whereby scar tissue replaces healthy liver parenchyma, leading to disruption of the liver architecture and hepatic dysfunction. Currently, there is no effective disease-modifying therapy for liver fibrosis. Recently, our group demonstrated that human umbilical cord blood (UCB) plasma possesses therapeutic effects in a rat model of acute liver failure.

Methods: In the current study, we tested whether exosomes (Exo) existed in UCB plasma and if they produced any antifibrotic benefits in a liver fibrosis model.

Results: Our results showed that UCB-Exo improved liver function and increased matrix metalloproteinase/tissue inhibitor of metalloproteinase degradation to reduce the degree of fibrosis. Moreover, UCB-Exo were found to suppress hepatic stellate cell (HSC) activity in vitro. These effects were associated with suppression of transforming growth factor- $\beta$ /inhibitor of DNA binding 1 signaling.

Conclusions: These results further support that UCB-Exo have antifibrotic effects in mice with liver fibrosis and activated HSCs and may herald a new cell-free antifibrotic therapy.
\end{abstract}

Keywords: Cirrhosis, Exosomes, Hepatic stellate cells, Inhibitor of DNA binding 1

\section{Introduction}

Liver fibrosis is a healing process associated with chronic inflammation. It may lead to cirrhosis and eventually can develop into cancer. Fibrogenesis is a vicious cycle driven by a number of damaging factors including inflammation and oxidative stress that underlie various liver conditions, such as hepatitis $\mathrm{B}$ and $\mathrm{C}$, alcoholic liver disease, non-alcoholic fatty liver disease (NAFLD), and autoimmune liver diseases [1]. Hepatic stellate cells (HSCs) play a central role in liver fibrosis. When parenchymal epithelial cells such as hepatocytes and cholangiocytes suffer chronic injury, they secrete inflammatory mediators to

\footnotetext{
*Correspondence: wyaoming@gmail.com

${ }^{1}$ Department of Surgery, National Taiwan University Hospital, Taipei, Taiwan

Full list of author information is available at the end of the article
}

promote HSC activation. This results in HSC proliferation and morphological transition to become myofibroblast-like cells, which elaborate excessive extracellular matrix (ECM) that accumulates in the liver to disrupt hepatic architecture and lead to liver dysfunction [2,3]. As a result, termination of this vicious cycle or elimination of fibrogenic HSCs may represent an approach for future antifibrotic therapies in the clinical setting of liver cirrhosis. During the vicious cycle, TGF- $\beta$ (transforming growth factor $\beta$ ), a profibrotic cytokine, contributes to liver fibrosis through phosphorylation of Smad 2 to activate HSC differentiation into myofibroblasts $[4,5]$. Therefore, enhanced TGF- $\beta$ signaling in liver fibrosis correlates with progression of fibrosis [6]. Recent research indicates that TGF- $\beta$ may interact with inhibitor of DNA binding protein (ID) 1-4-associated signaling pathways. ID belongs to the helix-loop-helix (HLH) protein family, 
which is responsible for cell cycle regulation and cell differentiation [7]. Of all the ID proteins, ID1 is linked to tumorigenesis, cellular senescence, and cell proliferation [8]. In addition, ID1 levels are highly expressed in hepatocellular carcinoma $[9,10]$. Therefore, inhibition of ID1 expression may be a promising therapeutic approach.

Stem cell therapy has been showing promising effects in regenerative medicine. In general, stem cells have the capacity to differentiate into diverse cell types to replace the damaged cells at a site of injury. Moreover, stem cells secrete biological factors to affect surrounding tissues [11]. Although stem cell therapy provides hope for degenerative diseases, there still exist some challenges, including host cell rejection, phenotypic stability, and tumor risk $[12,13]$. This calls for safe and effective cellfree therapies. Recent research has focused on exosomes as one such therapeutic agent. Exosomes are produced by various cells, such as stem cells, cancer cells, and immune cells. In addition, exosomes are present in various body fluids, including blood, urine, plasma, amniotic fluid, and semen. In fact, exosomes are responsible for cellto-cell communication and signal transmission, as well as facilitating repair of damaged tissues. Umbilical cord blood (UCB) contains mesenchymal stem cells (MSCs) and hematopoietic stem cells, and the proliferation and function of these stem cells are affected by a variety of cytokines, growth factors, and immunomodulatory agents $[14,15]$. These factors are reported to improve functional performance and reduce structural damage in a mouse model of acute ischemic stroke and have been suggested as potential cell-free therapeutic agents [16]. However, the therapeutic potential of UCB plasma has not been elucidated in liver diseases, and a recent study has shown that exosomes recapitulate the therapeutic effects of stem cell therapy $[17,18]$. Our previous research demonstrated that UCB plasma biomolecules decrease the damage in D-galactosamine (D-GalN)induced acute liver failure [19]. Hence, our current work aims to characterize UCB plasma-derived exosomes and demonstrate their potential as a new promising tool in the cell-free therapeutic armamentarium for the treatment of liver fibrosis.

\section{Materials and methods}

\section{Isolation and characterization of exosomes}

Human UCB and peripheral blood (PB) plasma were purchased from AllCells LLC (Alameda, CA), and exosomes were acquired using ExoQuick ${ }^{\mathrm{TM}}$ Exosome isolation reagent (System Bioscience Inc., Mountain View, CA). Briefly, plasma was mixed with the exosome isolation reagent and incubated at $4^{\circ} \mathrm{C}$ overnight. The mixture was centrifuged at $1500 \mathrm{~g}$ for $5 \mathrm{~min}$, then the supernatant was removed. The pellet was washed with PBS and centrifuged. Exosome fractions were characterized with CD63, CD9, CD81, and Hsp70 by the ExoAB Antibody kit (System Bioscience Inc.). Exosome sizes and concentrations were quantified using NanoSight (NanoSight Ltd., Amesbury, UK, Malvern. Com). Protein concentrations were determined by Pierce BCA assay (Thermo Scientific, Rockford, USA). The morphology of exosomes was determined by transmission electron microscopy (TEM). Exosomes were mounted on copper grids and were added $2 \%$ of uranyl acetate solution for $30 \mathrm{~s}$. The copper grids were then moved to the sample plate and stored in drying oven. HT-7700 Hitachi TEM was used to image exosome samples at a voltage of $100 \mathrm{kV}$. For exosome staining and uptake, exosomes were labeled with PKH-67 Green Fluorescent Cell Linker Kit for General Cell Membrane Labelling (Sigma) according to the manufacturer's protocol. Briefly, the exosomes were diluted in PBS and added to Diluent $C(1 \mathrm{~mL}$ Diluent $C$ with same volume of PBS as control), then $4 \mu \mathrm{L}$ of PKH-67 were added to it and incubated for $4 \mathrm{~min}$. This was followed by addition of $2 \mathrm{~mL}$ of $1 \%$ BSA (Sigma) to bind the excess dye. This was washed 4 times with $5 \mathrm{~mL}$ of PBS using a $300-\mathrm{kDa}$ filter (Microcon TM-300, Millipore) to remove excess dye, and then incubated with HSCs for $24 \mathrm{~h}$. Uptake efficiency was observed with flow cytometry. Exosome-free PBS receiving the same treatment was used as control.

\section{Protein extraction and gel electrophoresis}

The proteins were extracted with ExtractPRO ${ }^{\mathrm{TM}}$ Protein extraction reagent (Visual Protein, Taiwan). The protein was analyzed using 12.5\% SDS-PAGE. After electrophoresis, the gels were stained with VisPRO 5 min Protein Stain Kit (Visual Protein, Taiwan). The gel lanes corresponding to the samples were cut in 2 slices, and each slice was processed for in-gel digestion according to the method of Shevchenko. Briefly, slices were washed/rehydrated three times in $25 \mathrm{mM} \mathrm{ABC}$ (ammonium bicarbonate $\mathrm{pH} 7.9)+50 \% \mathrm{ACN}$ (acetonitrile) $/ 50 \mathrm{mM} \mathrm{ABC}$ (ammonium bicarbonate $\mathrm{pH}$ 7.9). Subsequently, proteins were reduced with $10 \mathrm{mM}$ dithiothreitol for $1 \mathrm{~h}$ at $56{ }^{\circ} \mathrm{C}$ and alkylated with $25 \mathrm{mM}$ iodoacetamide for $45 \mathrm{~min}$ at $24{ }^{\circ} \mathrm{C}$ in the dark. After two subsequent wash cycles, the slices were dried and incubated overnight with $20 \mathrm{ng} / \mu \mathrm{L}$ MS-grade Trypsin Gold (Promega, Madison, WI). Peptides were extracted three times in $10 \mu \mathrm{L}$ of $50 \% \mathrm{ACN}$ in $1 \%$ formic acid. The volume was dried out in a vacuum centrifuge prior to LC-MS/MS analysis.

\section{Nano-LC separation and mass spectrometry}

Peptides were separated using an Ultimate 3000 nanoLC system (Thermo Fisher Scientific, Bremen, Germany). Mobile phase A was $0.1 \%$ formic acid in water, and mobile phase B was composed of $100 \%$ acetonitrile with 
$0.1 \%$ formic acid. A segmented gradient in $90 \mathrm{~min}$ from 2 to $35 \%$ solvent $\mathrm{B}$ at a flow rate of $300 \mathrm{~nL} / \mathrm{min}$ and a column temperature of $35^{\circ} \mathrm{C}$ were used. Intact peptide mass spectra and fragmentation spectra were acquired on a Thermo Scientific ${ }^{\mathrm{TM}}$ Orbitrap Fusion ${ }^{\mathrm{TM}}$ Lumos $^{\mathrm{TM}}$ Tribrid $^{\mathrm{TM}}$ Mass Spectrometer (Thermo Fisher Scientific, UK). Mass spectrometry analysis was performed in a datadependent mode with Full-MS (externally calibrated to a mass accuracy of $<5 \mathrm{ppm}$, and a resolution of 120,000 at $m / z=200$ ) followed by HCD-MS/MS of the most intense ions in $3 \mathrm{~s}$. High-energy collision activated dissociation (HCD)-MS/MS (resolution of 15,000) was used to fragment multiply charged ions (charge state 2-7) within a 1.4 Da isolation window at a normalized collision energy of $32 \mathrm{eV}$.

\section{Bioinformatic analysis}

The accession number of each identified protein was loaded to the FunRich software V3.1.3 (http://www.funri ch.org) and mapped according to their Gene Ontology (GO), to determine their biological and functional properties. The identified proteins were compared with available exosome data from ExoCarta database (http://www. exocarta.org).

\section{Cells}

Mouse HSCs were isolated with density gradient centrifugation using C57BL/6 mice. Briefly, the mouse liver was perfused in situ with pronase and collagenase (Sigma), followed by Histodenz (8.3\%, Sigma) density gradient centrifugation. HSCs were maintained in Medium 199 (Sigma) with 10\% fetal bovine serum (FBS, Hyclone Laboratories Inc, USA), antibiotics, and L-glutamine (Gibco, Carlsbad, CA) at $37^{\circ} \mathrm{C}$ in a $5 \% \mathrm{CO}_{2}$ incubator. All experiments were performed at passages 2 to 7 . Human HSC line (LX2) was cultured in DMEM medium (Gibco, Carlsbad, CA) with $10 \%$ FBS, antibiotics, and L-glutamine at $37{ }^{\circ} \mathrm{C}$ in a $5 \% \mathrm{CO}_{2}$ incubator.

Cell proliferation assay was performed using the CyQUANT $^{\circledR}$ Cell proliferation assay kit (Invitrogen, USA). HSCs (5,000/well) were seeded in 96-well culture plates with Medium 199 containing 0.2\% Exo-free FBS, and treated with different concentrations of UCBExo $(10,20,40,80,160 \mu \mathrm{g} / \mathrm{mL})$. After $24 \mathrm{~h}$, the medium was removed, washed once with PBS, and then stored at $-80{ }^{\circ} \mathrm{C}$. On the next day, it was added to a detection reagent for $5 \mathrm{~min}$ and then read with Ex480/Em520. To determine LX2 cell proliferation, the LX2 cells were seeded in 96-well culture plates and added to $20 \mu \mathrm{g} / \mathrm{mL}$ $\left(\sim 5 \times 10^{6}\right.$ particles $)$ of UCB-Exo or PB-Exo with/without TGF- $\beta$ ( $5 \mathrm{ng} / \mathrm{mL})$. After $24 \mathrm{~h}$, cell proliferation was measured. For TGF- $\beta$ stimulation, LX2 cells were pretreated with UCB-Exo or PB-Exo for $24 \mathrm{~h}$, then stimulated by
TGF- $\beta$ ( $5 \mathrm{ng} / \mathrm{mL})$ for $0,0.5,1$, and $2 \mathrm{~h}$. Cells were then collected for western blotting.

\section{$\mathrm{CCl}_{4}$-induced liver fibrosis and UCB-Exo therapy}

C57BL/6 mice (7-week-old males) received intraperitoneal injections of carbon tetrachloride $\left(\mathrm{CCl}_{4}, 1 \mu \mathrm{g} / \mathrm{g}\right.$ body weight, prepared in olive oil, 1:5; Sigma) twice per week. After one month of $\mathrm{CCl}_{4}$ challenge, the mice were randomly divided into two groups ( $n=10$ /group). One group was infused with PBS, while the other group was infused with UCB-Exo $(250 \mu \mathrm{g} / 0.1 \mathrm{~mL})$ via a tail vein twice a week. Both groups received sustained administrations of $\mathrm{CCl}_{4}$. The mice were sacrificed for analysis of liver tissue and serum at the second and third months after $\mathrm{CCl}_{4}$ injection (Fig. 3A). All experimental procedures involving animals were approved by the Institutional Animal Care and Use Committee of National Taiwan University. All animals were purchased from National Laboratory Animal Center, Taipei, Taiwan.

\section{Liver enzyme, hepatic collagen content, and gelatinase activity measurements}

Serum aspartate aminotransferase (AST) and alanine aminotransferase (ALT) were measured with FUJI DRICHEM slide (FUJIFILM Drichen 4200, Japan). Hepatic collagen content was determined by Sircol Collagen Assay (Biocolor, Belfast, Northern Ireland). Collagen concentrations were calculated using a standard curve generated by acid-soluble type 1 collagen and expressed relative to total protein by Pierce BCA measurement (Thermo Scientific, Rockford, USA). For gelatinase (MMP-2 and MMP-9) activity measurements, InnoZymeTM Gelatinase (MMP-2/MMP-9) activity assay kit (Millipore) was used according to manufacturer's instructions.

\section{Histopathological and immunohistochemical staining}

Liver tissue was paraffin-embedded, processed into $5 \mu \mathrm{m}$ sections, and then stained with hematoxylin and eosin (H\&E) (Sigma) and Picro-Sirius red solution (ScyTek Laboratories, Logan, USA). Fibrosis score was quantified with reference to the Ishak fibrosis stage system [20]. The mean value of 10 randomly selected areas per mouse, magnification $\times 100$, was obtained. For immunohistochemical staining, liver sections were incubated with diluted primary antibodies against anti- $\alpha$ SMA (1:500; Sigma) and inhibitor of DNA binding 1 (ID1, 1:500; Santa Cruz Biotechnology, Inc, Oregon, USA), according to the manufacturer's instructions. The proteins were visualized by EnVision + Dual Link System-HRP (DAB +), and counter-stained with Mayer's Hematoxylin (Dako, Carpinteria, CA, USA). 


\section{Western blot}

Mouse liver tissue was homogenized in lysis buffer (Sigma) supplemented with proteinase inhibitor cocktail (Roche Diagnostics, Germany), boiled in SDS protein buffer (Protech, Taiwan), and separated by $10 \%$ of SDS-PAGE following transfer to PVDF membrane. Primary antibodies were targeting TGF- $\beta$, MMP-2, MMP9, MMP-13, TIMP-1, TIMP-2 (purchased from Abcam, Cambridge, MA), collagen type I, $\alpha$-SMA (Sigma), ID1 (Santa Cruz Biotechnology, Inc, Oregon, USA), Smad2/3, and phospho-Smad2/3 (Cell Signaling Transduction). Secondary antibodies were anti-rabbit and anti-mouse antibodies (IgG-HRP, Jackson ImmunoResearch, Dianova, Hamburg). Proteins were visualized by enhanced chemiluminescence detection (ECL, Millipore Corporation, Billerica, MA, USA). ImageJ software was used for quantification.

\section{Quantitative PCR}

Total RNA was extracted with TRIzol Reagent (Invitrogen, Carlsbad, CA, USA) using Direct-Zol RNA MiniPrep kit (Zymo Research, Orange, CA, USA). $1 \mu \mathrm{g}$ samples of RNA were reverse transcribed using a RevertAid ${ }^{\mathrm{TM}}$ $\mathrm{H}$ Minus First Strand cDNA Synthesis Kit (Fermentas, Thermo Scientific, Schwerte, Germany). RT-PCR was performed with Luminaris color HiGreen qPCR Master Mix (Fermentas, Thermo Scientific) on a CFX Connect Real-Time System (Bio-Rad, Hercules, CA, USA). The primer sequences are listed in Table 1.

\section{Statistical analysis}

Data are presented as mean \pm S.E.M. and are compared using two-tailed Student's $t$ test or one-way analysis of variance with Turkey's post hoc test used for multiple comparisons. Statistical significance is set to $p<0.05$.

\section{Results}

Isolation and identification of exosomes derived from UCB and PB

Our previous experiments showed that UCB contains a variety of cytokines with therapeutic effects in the acute liver failure rat model. Recently, exosomes have been shown to participate in cell communication and bring hope for therapeutic opportunities. Therefore, we believed that UCB may contain exosomes that could be used as therapeutic agents. First, we obtained the vesicles from UCB and PB with ExoQuick reagent. Nanoparticle tracking analysis showed that the size of the vesicles ranged from 52.0 to $126.0 \mathrm{~nm}$ in diameter and the median size of the vesicles was $81.1 \pm 2.4 \mathrm{~nm}$ for UCB-Exo (Fig. 1A). For PB-Exo, the size of vesicles ranged from 56.2 to $153.5 \mathrm{~nm}$ in diameter and the median size was $100.4 \pm 4.2 \mathrm{~nm}$ (Fig. 1A). They both were similar to the reported sizes of exosomes [21]. The UCB- and PB-Exo average particle count was $7.62 \times 10^{8}$ and $7.88 \times 10^{8}$ per $\mathrm{mL}$, respectively. The membrane integrity of exosomes was confirmed in the TEM image and appeared spherical in structure after isolation from UCB and PB (Fig. 1C). The particle sizes were consistent in samples isolated from UCB-Exo and PB-Exo under TEM. Next, to further confirm that our preparation contained exosomes, we used western blotting to detect four common exosome markers, including CD63, CD9, CD81, and HSP70. Our data clearly verify that UCB- and PB-Exo were positive for these exosome markers (Fig. 1B). These results indicated that exosomes exist in UCB and PB.

\section{Bioinformatic analysis of exosomes from UCB and PB}

Proteomic analysis of the different sources of exosomes revealed 1,011 and 555 proteins were present in UCBExo and PB-Exo, respectively. About $80 \%$ of UCB-Exo

Table 1 Primer sequences

\begin{tabular}{|c|c|c|}
\hline Target gene & Primer sequence $\left(5-3^{\prime}\right)$ & Amplicon size (bp) \\
\hline MMP-13 & $\begin{array}{l}\text { For: AACCCTAAGCACCCCAAAACA } \\
\text { Rev: GGTCAAAAACAGTTCAGGCTCAA }\end{array}$ & $150 \mathrm{bp}$ \\
\hline Pro-Collagen type I-a1 & $\begin{array}{l}\text { For: CCAGCGGTGGTTATGACTTCA } \\
\text { Rev: GCTGCGGATGTTCTCAATCTG }\end{array}$ & $167 \mathrm{bp}$ \\
\hline MMP-2 & $\begin{array}{l}\text { For: ACCATGCGGAAGCCAAGAT } \\
\text { Rev: GCCCGAGCAAAAGCATCAT }\end{array}$ & $155 \mathrm{bp}$ \\
\hline MMP-9 & $\begin{array}{l}\text { For: CCTACTGCTGGTCCTTCTG } \\
\text { Rev: GGCTTCCTCCGTGATTCG }\end{array}$ & $157 \mathrm{bp}$ \\
\hline TIMP-1 & $\begin{array}{l}\text { For: CATGGAAAGCCTCTGTGGAT } \\
\text { Rev: CTCAGAGTACGCCAGGGAAC }\end{array}$ & $132 \mathrm{bp}$ \\
\hline TIMP-2 & $\begin{array}{l}\text { For: CACAGACTTCAGCGAATGGA } \\
\text { Rev: CCAGCATGAGACCTCACAGA }\end{array}$ & $124 \mathrm{bp}$ \\
\hline TGF- $\beta$ & $\begin{array}{l}\text { For: TTGCCCTCTACAACCAACACAA } \\
\text { Rev: GGCTTGCGACCCACGTAGTA }\end{array}$ & $103 \mathrm{bp}$ \\
\hline
\end{tabular}




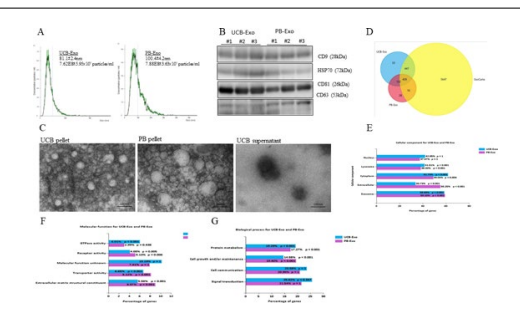

Fig. 1 Exosome identification and proteomic analysis in UCB-Exo and PB-Exo. A Size distribution of exosomes as measured by nanoparticle tracking analysis (NTA) showed that the UCB-Exo particle was $80.1 \mathrm{~nm}$ and concentration was $7.62 \times 108$ particles $/ \mathrm{mL}$, and the PB-Exo particle was $100.4 \mathrm{~nm}$ and concentration was $7.88 \times 108$ particles/ $\mathrm{mL}$. B Western blotting assay revealed that UCB-Exo and PB-Exo were positive for CD9, HSP70, CD81, and CD63. CTEM images of exosomes isolated from UCB and PB show spherically shaped particles. Bioinformatic analysis of UCB-EXo and PB-Exo. A Venn diagram of UCB-Exo and PB-Exo against ExoCarta (D). GO analysis for cellular components $(\mathbf{E})$, molecular function $(\mathbf{F})$, and biological processes of UCB-Exo and PB-Exo (G)

and PB-Exo proteins matched ExoCarta, indicating that the results were remarkably reliable (Fig. 1D). Comparing UCB-Exo and PB-Exo using a Venn diagram revealed 409 common proteins, and 53 and 28 proteins being exclusive to UCB-Exo and PB-Exo, respectively (Fig. 1D). GO analysis of the two source-derived exosomes showed that $60 \%$ of their component proteins could be correlated to available exosome data from ExoCarta. The exosomal proteins derived from UCB are more closely related to the proteins in the cytoplasm than the exosomal proteins derived from PB (Fig. 1E). In terms of molecular function, these proteins played significant roles in ECM, transporter activity, and GTPase activity (Fig. 1F). These proteins were also key participants in biological processes including protein metabolism and cell growth (Fig. 1G). These results suggest that plasma-derived exosomes have the potential for regeneration through these candidate proteins.

\section{UCB-Exo treatment inhibits HSC proliferation and reduces collagen production}

HSCs are major ECM producers in the liver, hence we prioritized testing whether UCB-Exo could be taken up by HSCs. Flow cytometry results showed that $78 \%$ of UCB-Exo labeled with PKH-67 were taken up by HSCs (Fig. 2A), and these were localized in the perinuclear region (Fig. 2B). Next, to evaluate the effect of UCB-Exo on cell proliferation of activated HSCs, activated HSCs were treated with different concentrations of UCB-Exo for $24 \mathrm{~h}$. Results showed that cell proliferation was significantly decreased starting from $10 \mu \mathrm{g} / \mathrm{mL}$ of UCB-Exo. Surprisingly, increasing UCB-Exo concentration further did not affect cell proliferation (Fig. 2C). Therefore, we chose the concentration of $20 \mu \mathrm{g} / \mathrm{mL}$ of UCB-Exo to perform further experiments. It is known that activated HSCs are the major cell type responsible for collagen production [22-24]. Therefore, we examined whether collagen production in HSCs would be affected by UCBExo. After treatment with $20 \mu \mathrm{g} / \mathrm{mL}$ of UCB-Exo for $24 \mathrm{~h}$, QPCR results showed that collagen $1 \alpha 2$ mRNA levels were significantly decreased in activated HSCs (Fig. 2D). In the meantime, measurements of culture media demonstrated a reduction in soluble collagen I content in activated HSCs treated with UCB-Exo (Fig. 2E). Moreover, ECM remodeling was related to MMP expression and associated with TGF- $\beta$ [25]. As shown in Fig. 2F, G, western blotting indicated that TGF- $\beta$ levels were significantly decreased in activated HSCs treated with UCBExo. In addition, MMP-2, MMP-9, and MMP-13 protein expressions were increased after UCB-Exo treatment for $72 \mathrm{~h}$ in activated HSCs. TIMP-1 and TIMP-2 expressions, which are inhibitors of MMP-9 and MMP-2, were not significantly different. Therefore, UCB-Exo treatment may significantly reduce cell proliferation, and enhance MMP levels to decrease collagen production.

\section{UCB-Exo improve liver function and decrease damage from $\mathrm{CCl}_{4}$}

Next, we investigated the therapeutic efficacy of UCBExo in liver fibrosis using the $\mathrm{CCl}_{4}$-induced liver fibrosis mouse model. The administration of $\mathrm{CCl}_{4}$ for 3 months caused severe liver damage as characterized by significantly elevated levels of serum AST ( 2 months: $372.4 \pm 70.6 \mathrm{U} / \mathrm{L} ; 3$ months: $3818.0 \pm 471.2 \mathrm{U} / \mathrm{L} ;$ Fig. $3 \mathrm{C})$ and ALT (2 months: $272.6 \pm 68.0 \mathrm{U} / \mathrm{L} ; 3$ months: $6524.0 \pm 928.7 \mathrm{U} / \mathrm{L}$; Fig. 3D), and as supported by the histopathological examination. H\&E staining demonstrated disruption of liver architecture, balloon cells, and inflammatory cell infiltration after 3 months of $\mathrm{CCl}_{4}$ exposure (Fig. 3B). However, no mice died during this experiment (prior to sacrifice). After receiving $\mathrm{CCl}_{4}$ for 1 month, the mice were infused with UCB-Exo 4 times (once a week). Histopathological examination (Fig. 3B) and serum levels of AST and ALT (Fig. 3C, D) showed no significant change when compared to the $\mathrm{CCl}_{4}$ groups. However, when receiving 8 infusions of UCB-Exo, the results showed that AST $(1472.5 \pm 271.9 \mathrm{U} / \mathrm{L}$; Fig. $3 \mathrm{C})$ and ALT $(2651.3 \pm 364.5 \mathrm{U} / \mathrm{L}$; Fig. 3D) levels were significantly decreased compared to the group exposed to $\mathrm{CCl}_{4}$ for 3 months. Altogether, incidence and severity of histopathological lesions were reduced in the group receiving UCB-Exo infusion for 8 times, but these were not significantly different between the 4 times group and the $\mathrm{CCl}_{4}$ group (Fig. 3B).

Subsequently, we investigated whether UCB-Exo could decrease collagen production. Sirius red staining 

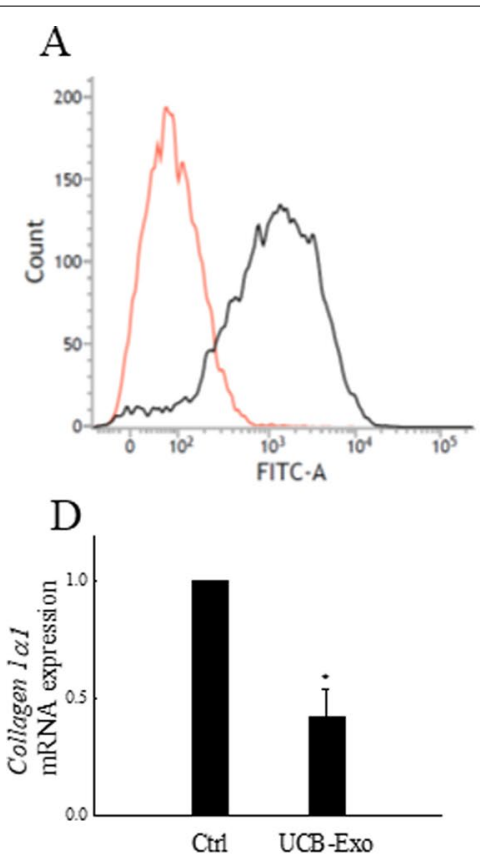

$\mathrm{E}$

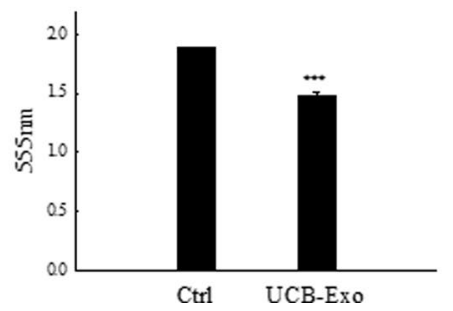

B

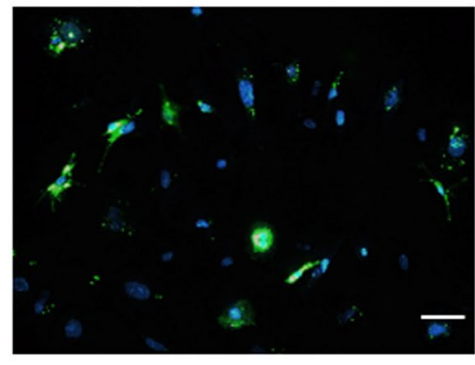

F

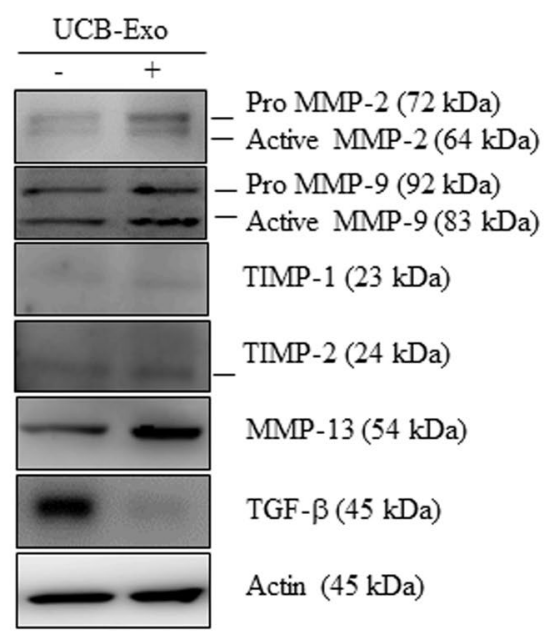

$\mathrm{C}$

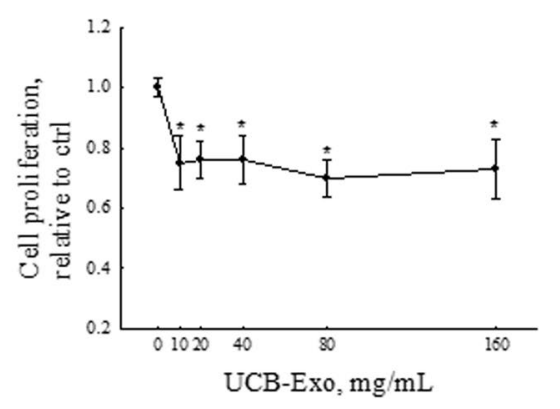

G

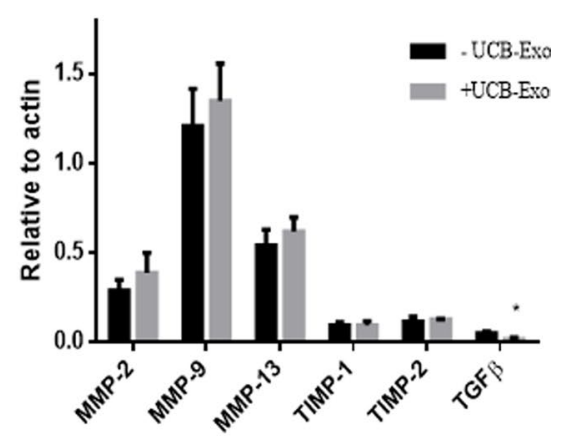

Fig. 2 UCB-Exo inhibit primary HSC activation and collagen production. Primary HSCs were incubated with PKH-labeled exosomes $(20 \mu \mathrm{g} / \mathrm{mL})$ for $12 \mathrm{~h}$ and analyzed by flow cytometry (A) and microscopy (B). Blue: DAPI; Green: PKH-labeled UCB-Exo. Scale bar: 50 um. HSCs were exposed to different concentrations of UCB-Exo for $24 \mathrm{~h}$ and cell proliferation is presented in (C). qRT-PCR analyses of collagen $1 \mathrm{a} 1$ expression in treatment with UCB-Exo for $24 \mathrm{~h}$ (D). Soluble collagen I content was measured in culture media after $24 \mathrm{~h}$ of UCB-Exo treatment (E). Western blotting analysis of MMP-2, MMP-9, MMP-13, TIMP-1, TIMP-2, and TGF- $\beta$ expression after $72 \mathrm{~h}$ of UCB-Exo treatment (F). Quantification of western blot results was performed by calculating the ratio of the value to that of actin. Data are presented as the mean \pm SEM. ${ }^{*} p<0.05$ and ${ }^{* * *} p<0.001$

may be used to visualize the collagen network and determine the degree of fibrosis in tissue. As Fig. 4A shows, the fibrotic area was significantly decreased in the liver that was infused with UCB-Exo for 8 times. Based on the results of Sirius red staining, we referred to the Ishak fibrosis grading system [26] to determine the histological fibrosis score ranging from 0 (no fibrosis) to 6 (cirrhosis). The average fibrotic scores were significantly lower in the UCB-Exo group compared to the $\mathrm{CCl}_{4}$ group $(3.88 \pm 0.29$ vs. $5.6 \pm 0.55)$ (Fig. $4 \mathrm{C}$ ). In addition, soluble collagen content was significantly lower in the liver tissue of UCB-Exo mice compared to $\mathrm{CCl}_{4}$ mice at 3 months (Fig. 4E). Examination of collagen expression using QPCR (Fig. 4F) and western blotting (Fig. 4G, H) revealed that levels of pro-collagen
mRNA and protein levels were significantly decreased after 8 times of UCB-Exo administration. Moreover, activated HSCs were not only responsible for collagen production, but also highly expressed $\alpha$-SMA and secreted TGF- $\beta$. Immunohistochemical analysis of $\alpha$-SMA showed that the group infused with UCB-Exo 8 times possessed significantly less $\alpha$-SMA positive areas than the $\mathrm{CCl}_{4}$ group, with the former group demonstrating diminished fibrotic networks (Fig. 4B). Further results showed that expression of $\alpha$-SMA protein was reduced in the 8-time UCB-Exo infusion group. QPCR assay (Fig. 4D) and western blotting (Fig. 4G, H) indicated that TGF- $\beta$ expression could be significantly lower in 4-time UCB-Exo infusion group compared to the $\mathrm{CCl}_{4}$ group at 2 months. Therefore, these results imply that UCB-Exo may exert an antifibrotic effect by decreasing collagen deposition. 
A

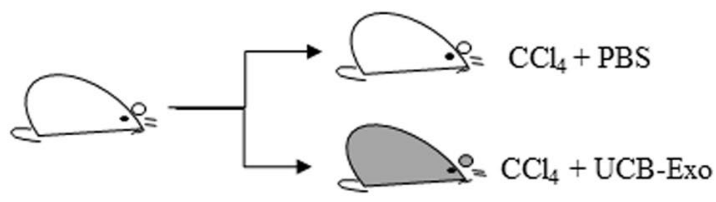

Time

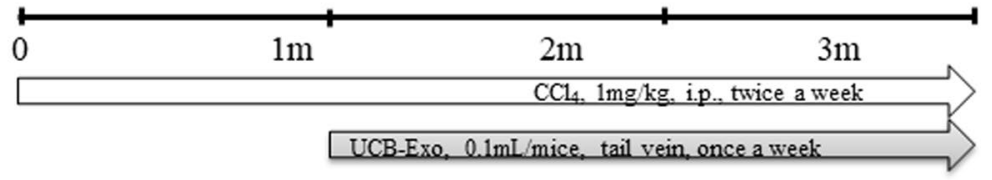

B

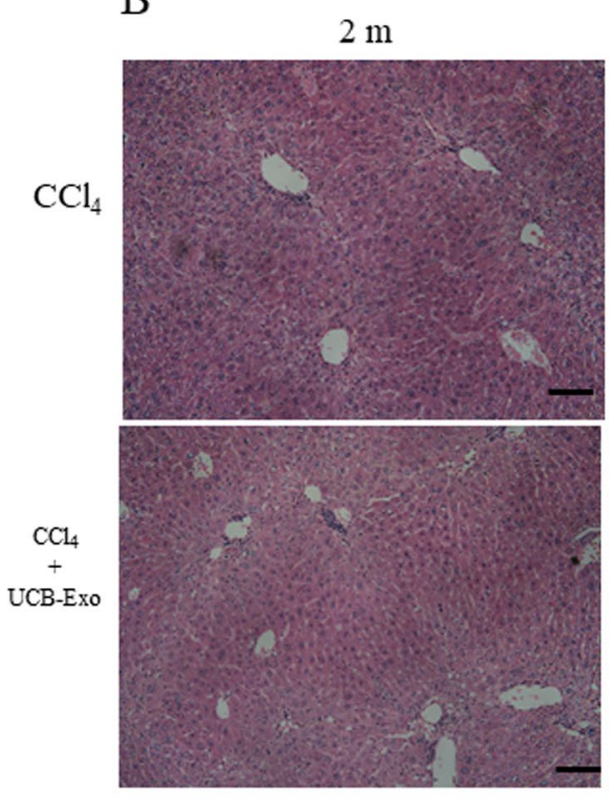

$3 \mathrm{~m}$

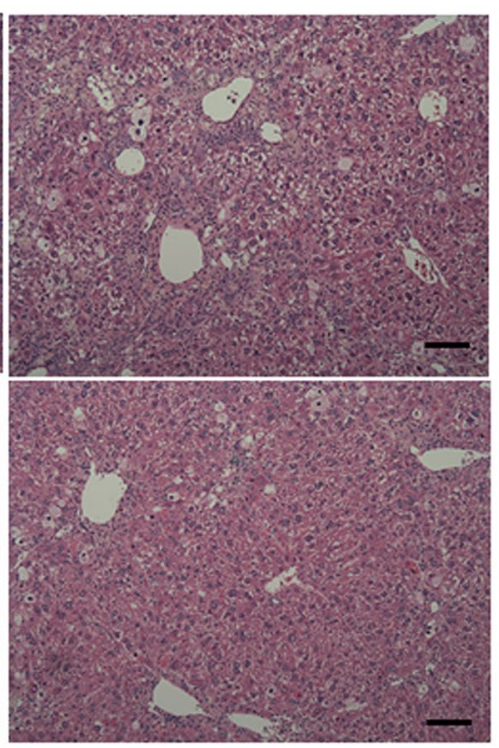

C
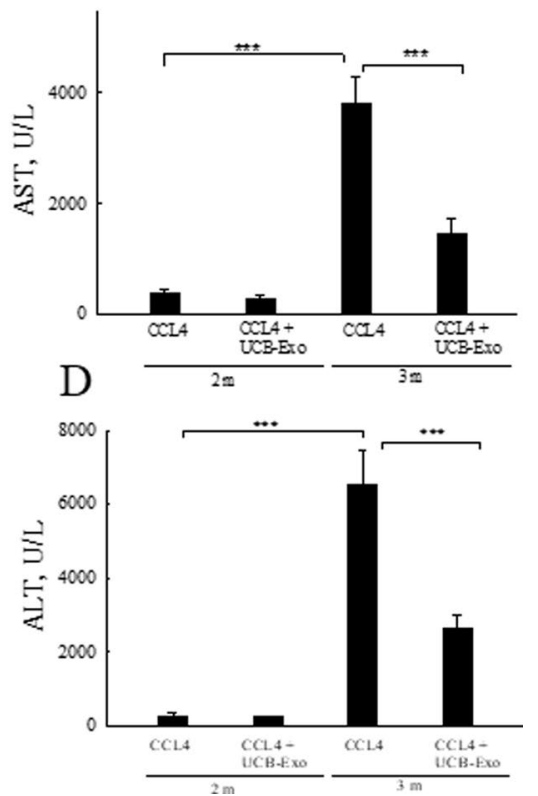

Fig. 3 UCB-Exo reduce histological injuries and improve liver function in mice with $\mathrm{CCl}_{4}$-induced liver fibrosis. Schematic diagram of liver fibrosis. Mice were administered $\mathrm{CCl}_{4}$ for 1 month $(n=20)$ and divided into two groups, namely, a $\mathrm{CCl}_{4}$ group $\left(\mathrm{CCl}_{4}+\mathrm{PBS}\right)$, and an UCB-Exo group $\left(\mathrm{CCl}_{4}+\right.$ UCB-Exo for 2 months) (A). Liver sections were stained with H\&E and are shown in (B). Scale bar: $100 \mu \mathrm{m}$. Serum AST (C) and ALT (D) levels were analyzed. Data are presented as the mean \pm SEM. ${ }^{* *} p<0.001$

\section{UCB-Exo exert antifibrotic effects in the mouse model of liver fibrosis}

During the fibrogenesis, activated HSCs produce ECM that accumulate in the liver. ECM homeostasis is regulated by the balance of MMPs and TIMPs, which is important in tissue remodeling and liver fibrogenesis. MMP-2 is responsible for turnover of ECM and tissue homeostasis, whereas MMP-9 is involved in tissue injury and fibrogenesis. Their activity is inhibited by TIMP-2 and TIMP-1, respectively [27]. We measured MMP-2, MMP-9, and their inhibitors (TIMP1, TIMP-2) in the $\mathrm{CCl}_{4}$-induced liver fibrosis group with/without UCB-Exo treatment. Figure 5 shows that mRNA and protein expression of MMP-2 and MMP-9 were significantly higher in the UCB-Exo 8-time infusion group (Fig. 5A, B). Although TIMP-1 mRNA was increased in the 8-time UCB-Exo infusion group, this did not reach statistical significance (Fig. 5C), whereas protein expression of TIMP-1 was inhibited by treatment with UCB-Exo for 8 times (Fig. 5E, H). Similarly, TIMP-2 mRNA and protein levels were also significantly lower (Fig. 5D, E, H). Besides, the activity of MMP-2/9 was slightly increased in the UCB-Exo group in comparison with the $\mathrm{CCl}_{4}$ group (Fig. 5I, 1.57 $\pm 0.08 \mathrm{vs} 2.0 \pm 0.10 \mathrm{ng} / \mu \mathrm{g}$ protein). The net activity of ECM turnover or fibrosis regression would depend on the balance between MMPs and TIMPs [28]. Therefore, we performed quantitative analysis of western 


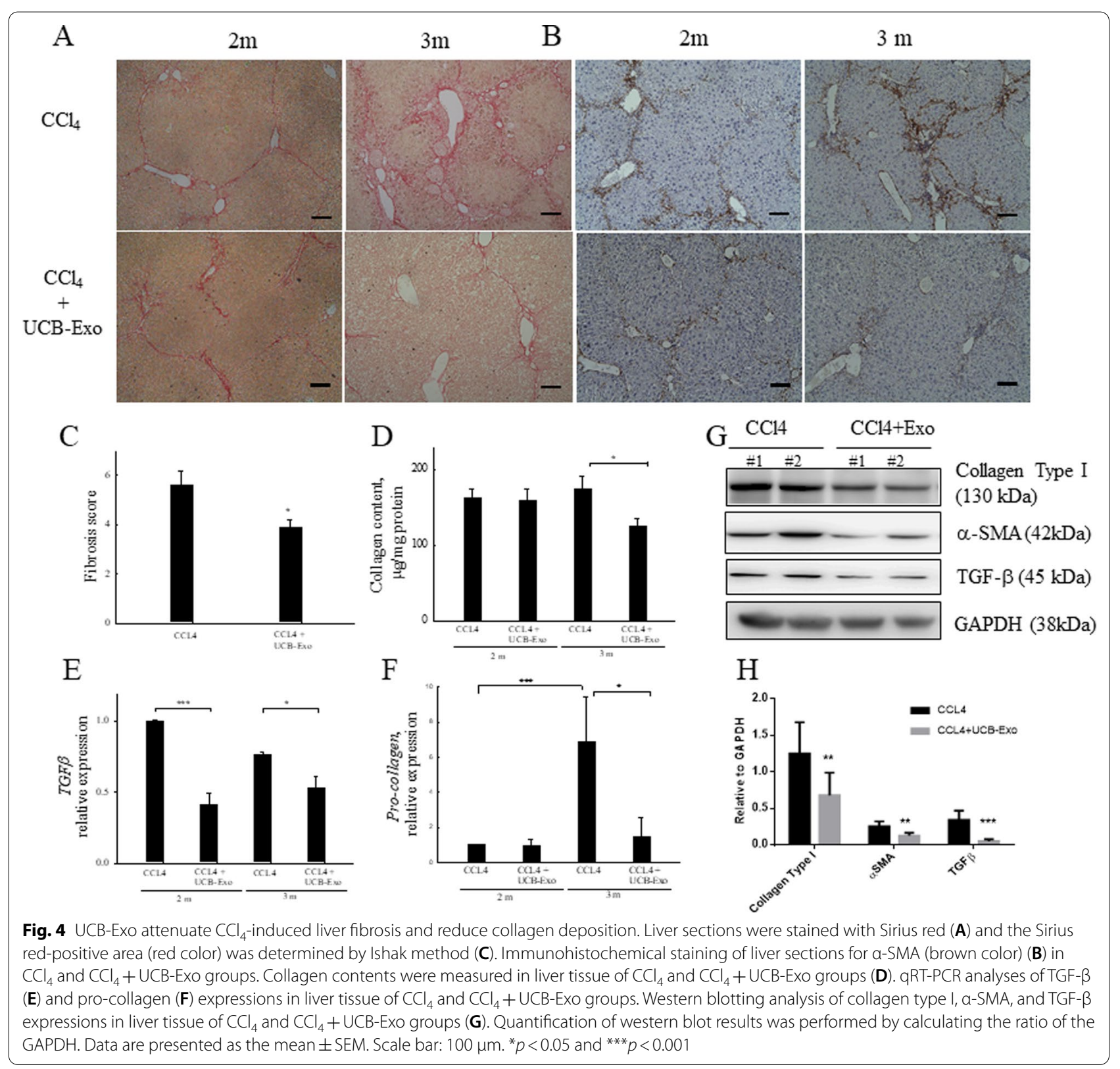

blot results and assessed the ratios of MMP-2/TIMP-2 and MMP-9/TIMP-1. Our results indicated a threefold increase in proteolytic activity for the UCB-Exo group in comparison with the $\mathrm{CCl}_{4}$ group (Fig. 5F, G). These results revealed that UCB-Exo treatment tilted the MMP/TIMP balance against fibrogenesis.

\section{UCB-Exo treatment hinders HSC activity through inhibition of the TGF- $\beta$-ID1 signaling pathway both in vivo and in vitro.}

Typically, TGF- $\beta$ is a master pro-fibrogenic cytokine that is associated with HSC activation and liver fibrosis
[29]. Here, we studied whether UCB-Exo and PB-Exo exerted protective effects in LX2 cells stimulated by TGF- $\beta$. Our data showed that cell proliferation was significantly increased by 1.68 -fold when treated with $5 \mathrm{ng} /$ $\mathrm{mL}$ of TGF- $\beta$ for $24 \mathrm{~h}$. Furthermore, cell proliferation was decreased by 1.12-fold if treated with UCB-Exo. In contrast, with PB-Exo treatment, cell proliferation was increased by 1.95 -fold (Fig. 6A). Thus, cell proliferation appeared to be suppressed by UCB-Exo treatment, but not by PB-Exo. To understand the response to TGF- $\beta$ downstream, we pretreated LX2 cells with UCB-Exo or PB-Exo for $24 \mathrm{~h}$, followed by stimulation with TGF- $\beta$ 


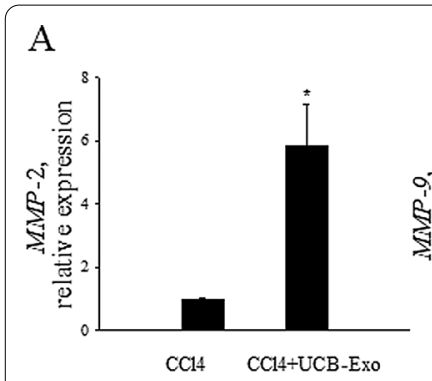

$\mathrm{E}$

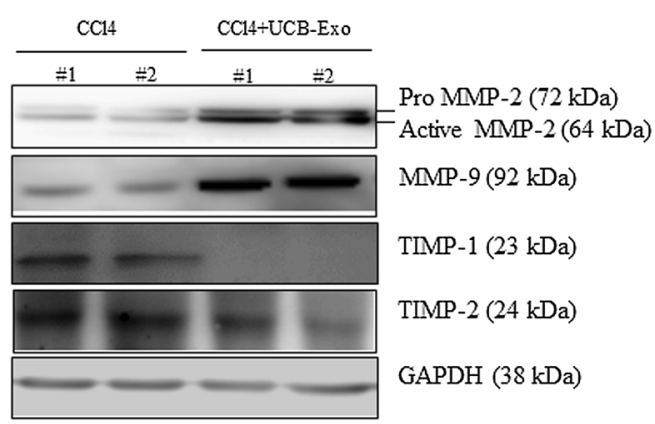

$\mathrm{C}$

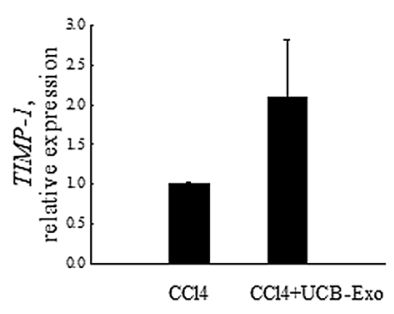

$\mathrm{F}$
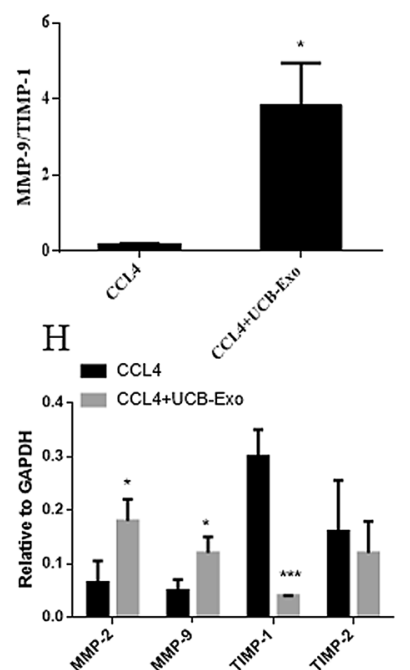

$\mathrm{D}$

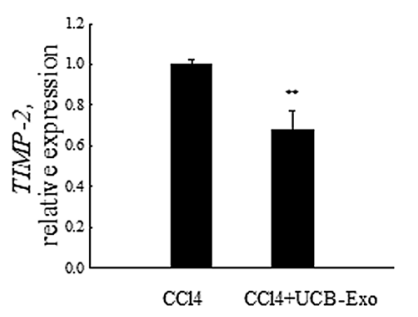

G
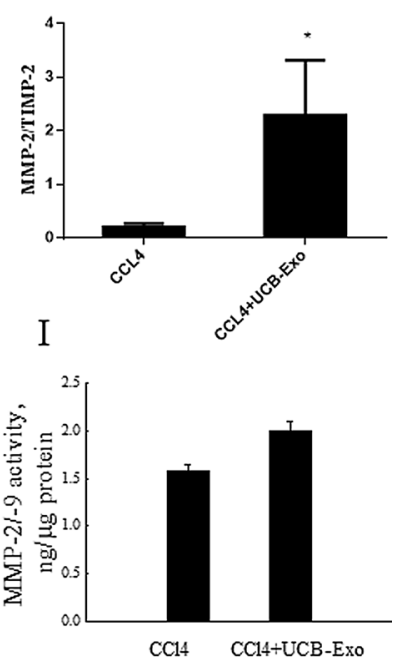

Fig. 5 UCB-Exo increase MMP degradation in CCl -induced liver fibrosis. qRT-PCR analyses of MMP-2 (A), MMP-9 (B), TIMP-1 (C), and TIMP-2 (D) expressions after 2 months of UCB-Exo treatment and in the $\mathrm{CCl}_{4}$ group. Western blotting assay for MMP-2, MMP-9, TIMP-1, and TIMP-2 in the liver tissue of $\mathrm{CCl}_{4}$ and $\mathrm{CCl}_{4}+\mathrm{UCB}$-Exo groups (E). Quantification of western blot results was performed by calculating the ratio of MMP-9 to TIMP-1 $(\mathbf{F})$, the ratio of MMP-2 to TIMP-2 $(\mathbf{G})$, and the ratio of the value to GAPDH $(\mathbf{H})$. MMP-2 and MMP-9 activities were increased in the liver tissue of the $\mathrm{CCl}_{4}+\mathrm{UCB}$-Exo group compared to the $\mathrm{CCl}_{4}$ group in the third month (I). Data are presented as the mean $\pm \mathrm{SEM}$. ${ }^{*} p<0.05,{ }^{* *} p<0.01$ and ${ }^{* * *} p<0.001$

for different durations $(0,0.5,1$, and $2 \mathrm{~h})$. Western blotting showed that TGF- $\beta$ stimulation could activate phospho-Smad $2 / 3$ expression depending on the duration of TGF- $\beta$ exposure, with a significant increase in expression observed at $2 \mathrm{~h}$ duration. As Fig. 6B shows, UCB-Exo and PB-Exo slightly suppressed phospho-Smad2/3 expression in the TGF- $\beta$ stimulation condition. ID1 protein expression was significantly induced by TGF- $\beta$ stimulation in LX2 cells, and UCB-Exo was found to inhibit ID-1 expression substantially. Although there seemed to be a trend that UCB-Exo reduced pSmad2/3 and ID1, there was no significant difference between treatment with UCB-Exo or PB-Exo (Fig. 6C, D). Based on the verified correlation between ID1 and TGF- $\beta$ in LX2 cells, we further detected the expression of ID1 in the liver fibrosis mouse model. Immunohistochemical analysis revealed that ID1 was localized to the cell cytoplasm, and it was strongly expressed in the liver sections exposed to $\mathrm{CCl}_{4}$.
After infusion of UCB-Exo for 4 times, ID1 expression did not change significantly in comparison with the control group after exposure to $\mathrm{CCl}_{4}$ for 2 months. After UCB-Exo infusion for 8 times, however, ID1 expression was indeed significantly decreased compared with the control group after $\mathrm{CCl}_{4}$ exposure for 3 months (Fig. 6E). Overall, these results showed that UCB-Exo downregulated TGF- $\beta /$ ID1 signaling and their therapeutic antifibrotic effect was superior to that of PB-Exo.

\section{Discussion}

Liver fibrosis is irreversible and this passive process leads to progressive cirrhosis and liver failure. However, the molecular and cellular mechanisms of liver fibrosis continue to be developed. Currently, no specific antifibrotic options are available to prevent or cure liver cirrhosis. Thus, it is necessary to develop a safe, effective, and clinically viable strategy for prevention of hepatic fibrogenesis 


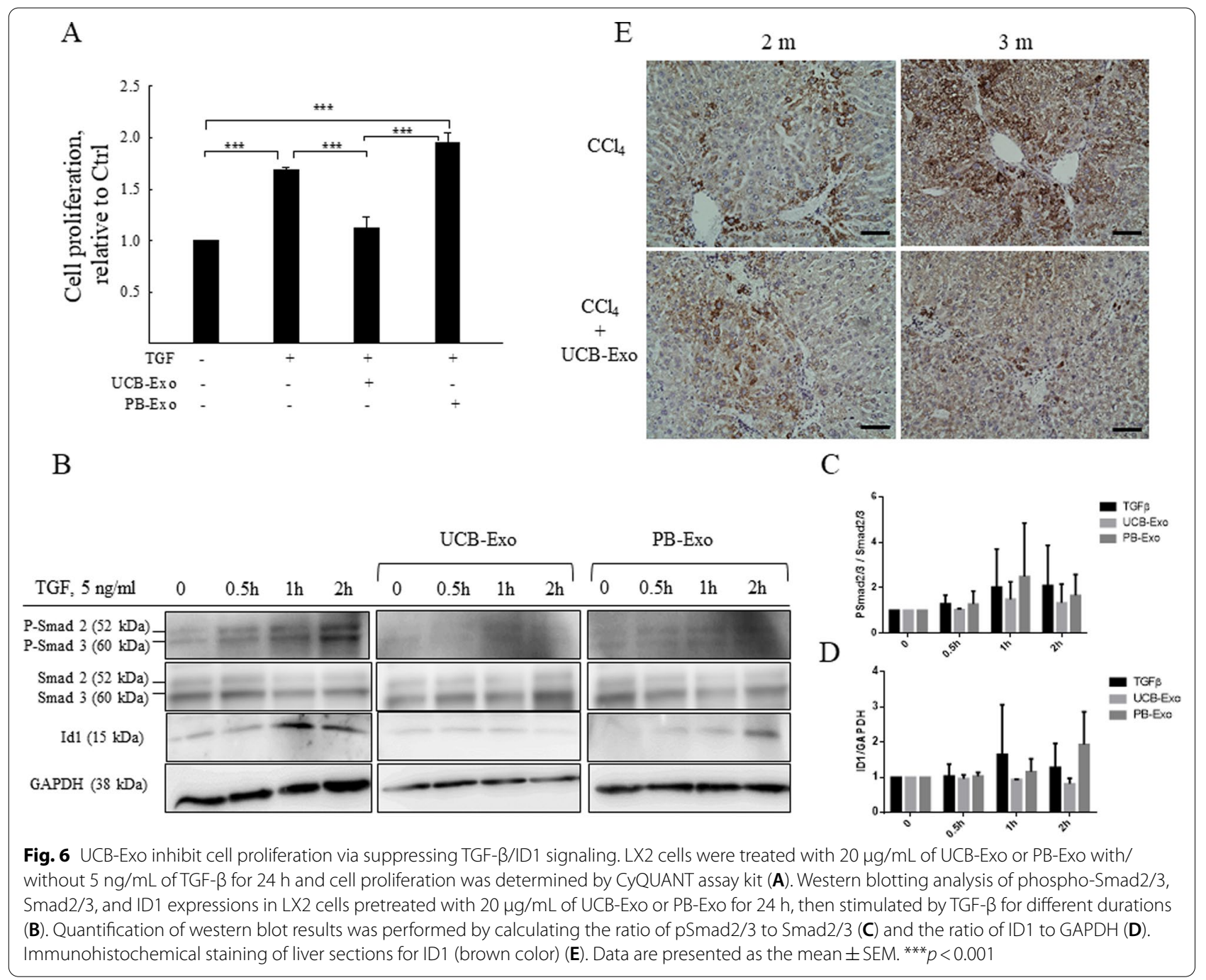

and improvement of hepatic function. Recent reports described that exosomes may provide a therapeutic opportunity, due to them playing important roles in paracrine and autocrine signaling [30]. In this study, antifibrotic effects of UCB-Exo and the potential mechanisms were identified. Liver fibrosis in the mouse model was induced by $\mathrm{CCl}_{4}$ administration. Histopathological and molecular biological changes started at 2 months and continued to develop. It was shown that eight infusions of UCB-Exo mitigated some of these changes. Additionally, UCB-Exo inhibited HSC proliferation, and increased MMP/TIMP degradation to reduce collagen production, mediated via downregulation of the TGF- $\beta$-ID1 signaling pathway (Fig. 7). Our findings may underpin future antifibrotic therapies to prevent the progression of liver fibrosis and improve liver function.

Exosomes are considered to enhance or improve liver function and regeneration [31,32]. They act as messengers of intercellular communication and regulate the biological properties of recipient cells [33-35]. Exosomes originate from various types of cells and carry different therapeutic values. Currently, a major source of exosomes is MSCs, found in bone marrow, umbilical cord blood, and adipose tissue [36, 37]. They are able to attenuate liver fibrosis [38]. Compared to cell-based therapies, exosomes are powerful carriers that move across biological barriers $[39,40]$. In addition, no immune response has been reported in patients undergoing MSCExo therapy [41]. Further advantages of exosome therapy include reduction of tumor risk and ease of storage, with the literature suggesting that MSC-Exo inhibit tumor growth [42, 43]. Even though exosome therapy possesses multiple advantages over cell-based therapies, there still exist some challenges. Before application of MSC-Exo, MSCs must be acquired and incubated, and the quality of cells and exosomes are affected by the period of culture. 


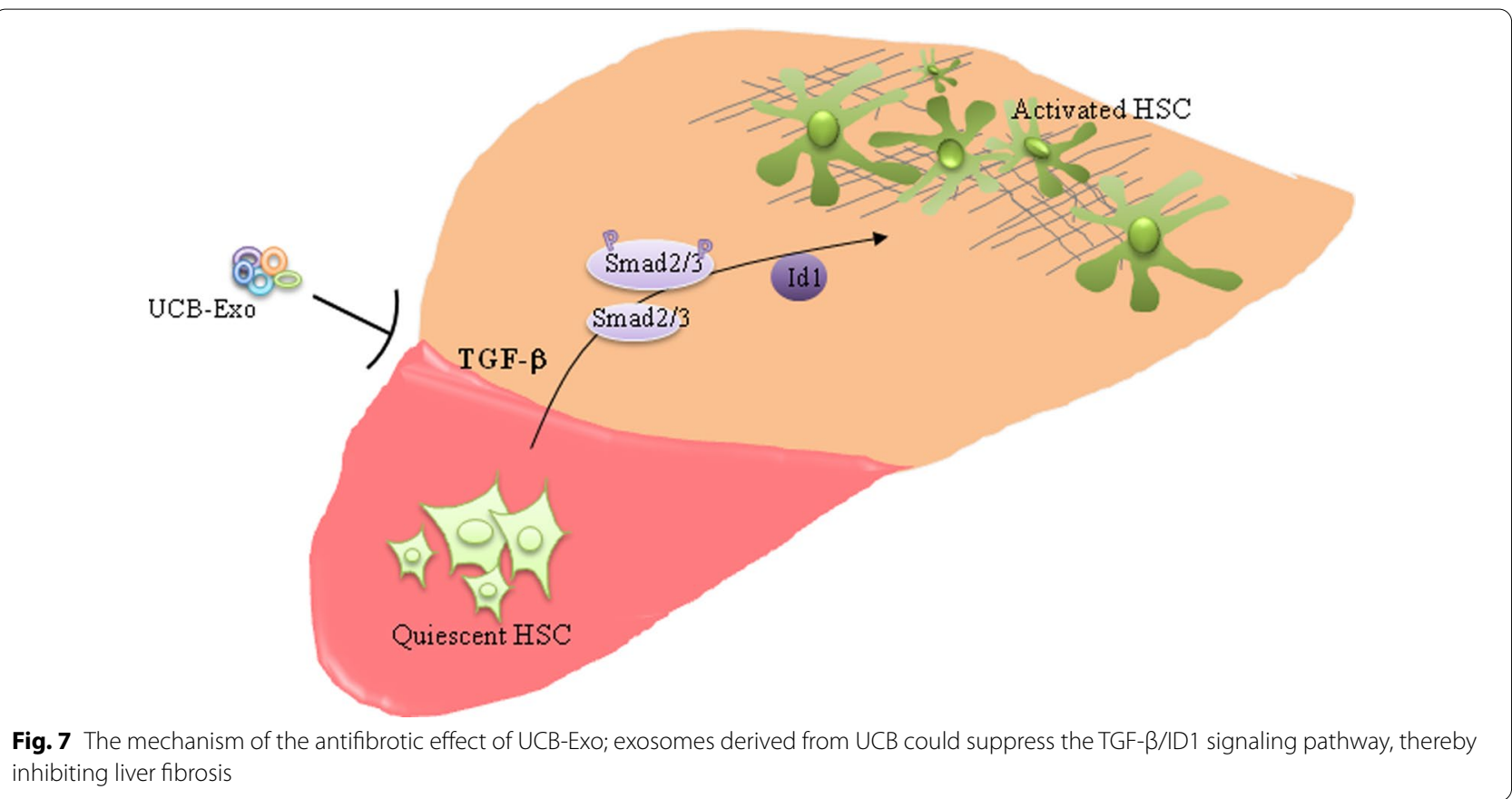

Therefore, using the exosomes derived from UCB plasma could avoid the time required for the culture of MSCs and be utilized immediately. In physiology, UCB plasma regulates the proliferation and function of MSCs, and represents a reservoir of growth factors, cytokines, and immunomodulatory mediators, whose concentrations are greater than that in MSC condition media [14]. Moreover, UCB plasma can be a FBS replacement for culturing MSCs [44], and may be available from a large number of high-quality cord blood banks worldwide. Hence, it may represent a novel compelling cell-free therapeutic option. In the present study, we identified that exosomes exist in UCB plasma and possess antifibrotic properties in diminishing collagen deposition in liver fibrogenesis. UCB plasma has been shown to improve functional performance and reduce structural damage in ischemic brain injury in rats [16]. Another study showed that UCB plasma improved hippocampal function in aged mice [45]. Previous findings also suggested that UCB plasma enhances liver function and reduces inflammation in rats with acute liver failure. In the present study, our results demonstrated that exosomes are present in both UCB and PB plasma. Nevertheless, bioinformatic analysis showed that their protein compositions differ. In vitro cell proliferation assay showed that UCB-Exo could inhibit HSC proliferation, in contrast to PB-Exo, which instead increased HSC proliferation. Therefore, UCB-Exo exerted more desirable effects in liver fibrosis.

TGF- $\beta$ is a crucial mediator for HSC activation and ECM accumulation in liver fibrogenesis [46, 47]. In our study,
TGF- $\beta$ expression was reduced in mice with liver fibrosis treated with UCB-Exo, suggesting that the antifibrotic effects of UCB-Exo may be mediated through the suppression of TGF- $\beta$. In addition, TGF- $\beta$ is a profibrotic factor that increases $\alpha$-SMA expression and collagen I production in HSCs [4, 48]. UCB-Exo treatment could diminish $\alpha$-SMA expression, and cell proliferation was reduced with in vitro TGF- $\beta$ stimulation, suggesting that UCB-Exo decreased HSC activation in vitro and in vivo. Moreover, TGF- $\beta$ signaling induces liver fibrosis through the sequential activation of downstream mediators including Smad2 and Smad3 [46, 47]. In this study, phospho-Smad2/3 was significantly increased after TGF- $\beta$ stimulation. UCBExo pretreatment inhibited the expression of phosphoSmad2/3 after TGF- $\beta$ stimulation. Furthermore, ID1 is one of the downstream effectors in TGF- $\beta$ signaling and participates in cell differentiation and the cell cycle. Several reports have provided evidence that ID1 expression is associated with TGF- $\beta$ expression [49-51]. Our results demonstrated that ID1 expression was significantly increased after TGF- $\beta$ stimulation, but more importantly, UCB-Exo pretreatment was able to suppress this effect while PBExo pretreatment could not. Thus, our study highlights a key role for UCB-Exo in antifibrotic applications for liver fibrosis. A weakness of our study is that we did not collect short-term evaluation data after UCB-Exo treatment, and we did not use surface marker analysis to clearly identify the exosomes. The other limitation of this study is that proteomic analysis of exosomes cannot exclude the possibility 
of contamination from unexpected molecules pulled down together during extraction.

In conclusion, our findings suggest that UCB-Exo ameliorate $\mathrm{CCl}_{4}$-induced liver fibrosis. UCB-Exo therapy significantly decreases collagen production under both in vitro and in vivo conditions. Inhibition of HSC proliferation and subsequent events reduce liver fibrosis. Moreover, UCBExo treatment can mitigate liver fibrosis through inhibition of the TGF- $\beta$ /ID1 signaling pathway. This study provides a novel mechanism for UCB-Exo regulated tissue repair and highlights an alternative source of exosomes in antifibrotic applications.

\section{Abbreviations}

UCB: Umbilical cord blood; PB: Peripheral blood; $\mathrm{CCl}_{4}$ : Carbon tetrachloride; Exo: Exosome; MMP: Metalloproteinase; TIMP: Tissue inhibitor of metalloproteinase; HSC: Hepatic stellate cell; TGF- $\beta$ : Transforming growth factor- $\beta$; ID1: Inhibitor of DNA binding 1; ECM: Extracellular matrix; D-GalN: D-galactosamine; AST: Aspartate aminotransferase; ALT: Alanine aminotransferase.

\section{Acknowledgements}

We thank the 6th and 7th Core Facility at National Taiwan University Hospital for providing technical services.

\section{Authors' contributions}

$\mathrm{Y}-\mathrm{JH}$ designed the study and performed the experiments. JC reviewed and edited the manuscript. C-YL helped perform the analysis with constructive discussion. Y-MW supported research funding. All authors read and approved the manuscript.

\section{Funding}

This work was supported by a grant from the Ministry of Science and Technology (MOST) of Taiwan 104-2314-B-002-227-MY3, 109-2314-B-002-079 and National Taiwan University Hospital 109-\$4585.

\section{Availability of data and materials}

All data generated or analyzed during this study are included in this published article.

\section{Declarations}

Ethics approval and consent to participate

Not applicable for that section.

\section{Consent for publication}

Not applicable for that section.

\section{Competing interests}

The authors declare that they have no competing interests.

\section{Author details}

${ }^{1}$ Department of Surgery, National Taiwan University Hospital, Taipei, Taiwan. ${ }^{2}$ Department of Surgery, Wollongong Hospital, Loftus Street, Wollongong, NSW 2500, Australia. ${ }^{3}$ Department of Surgery, National Taiwan University Hospital and National Taiwan University College of Medicine, No. 7, Chung-Shan South Road, Taipei, Taiwan.

Received: 11 July 2021 Accepted: 28 October 2021

Published online: 12 November 2021

\section{References}

1. Parola M, Pinzani M. Liver fibrosis: pathophysiology, pathogenetic targets and clinical issues. Mol Asp Med. 2019:65:37-55.

2. Higashi T, Friedman SL, Hoshida Y. Hepatic stellate cells as key target in liver fibrosis. Adv Drug Deliv Rev. 2017;121:27-42.

3. Friedman SL. Hepatic stellate cells: protean, multifunctional, and enigmatic cells of the liver. Physiol Rev. 2008;88(1):125-72.

4. Dooley $\mathrm{S}$, et al. Transforming growth factor beta signal transduction in hepatic stellate cells via Smad2/3 phosphorylation, a pathway that is abrogated during in vitro progression to myofibroblasts. TGFbeta signal transduction during transdifferentiation of hepatic stellate cells. FEBS Lett. 2001;502(1-2):4-10.

5. Budi EH, et al. TGF- $\beta$ as a driver of fibrosis: physiological roles and therapeutic opportunities. J Pathol. 2021;254(4):358-73.

6. Ghafoory S, et al. Platelet TGF- $\beta 1$ deficiency decreases liver fibrosis in a mouse model of liver injury. Blood Adv. 2018;2(5):470-80.

7. Zhao Z, et al. Inhibitor of differentiation 1 (Id 1) in cancer and cancer therapy. Int J Med Sci. 2020;17(8):995-1005.

8. Di K, et al. Id-1 modulates senescence and TGF-beta1 sensitivity in prostate epithelial cells. Biol Cell. 2006;98(9):523-33.

9. Matsuda Y, et al. Overexpressed Id-1 is associated with a high risk of hepatocellular carcinoma development in patients with cirrhosis without transcriptional repression of p16. Cancer. 2005;104(5):1037-44.

10. Roschger $C$, Cabrele $C$. The Id-protein family in developmental and cancer-associated pathways. Cell Commun Signal. 2017;15(1):7.

11. Caplan Al, Dennis JE. Mesenchymal stem cells as trophic mediators. J Cell Biochem. 2006;98(5):1076-84.

12. Musiał-Wysocka A, Kot M, Majka M. The pros and cons of mesenchymal stem cell-based therapies. Cell Transplant. 2019;28(7):801-12.

13. Martin U. Therapeutic application of pluripotent stem cells: challenges and risks. Front Med. 2017. https://doi.org/10.3389/fmed.2017.00229.

14. Pereira T, et al. MSCs conditioned media and umbilical cord blood plasma metabolomics and composition. PLoS ONE. 2014;9(11):e113769.

15. Phadnis SM, et al. Human umbilical cord blood serum promotes growth, proliferation, as well as differentiation of human bone marrow-derived progenitor cells. In Vitro Cell Dev Biol Anim. 2006;42(10):283-6.

16. Yoo J, et al. Therapeutic effects of umbilical cord blood plasma in a rat model of acute ischemic stroke. Oncotarget. 2016;7(48):79131-40.

17. Park KS, et al. Enhancement of therapeutic potential of mesenchymal stem cell-derived extracellular vesicles. Stem Cell Res Ther. 2019;10(1):019-1398

18. Keshtkar S, Azarpira N, Ghahremani MH. Mesenchymal stem cell-derived extracellular vesicles: novel frontiers in regenerative medicine. Stem Cell Res Ther. 2018;9(1):018-0791.

19. Huang YJ, et al. Therapeutic potential of plasma proteins derived from umbilical cord blood for acute liver failure. Mol Pharm. 2019:16(3):1092-104.

20. Ishak K, et al. Histological grading and staging of chronic hepatitis. J Hepatol. 1995:22(6):696-9.

21. Kastelowitz N, Yin H. Exosomes and microvesicles: identification and targeting by particle size and lipid chemical probes. ChemBioChem. 2014;15(7):923-8

22. Geerts A. History, heterogeneity, developmental biology, and functions of quiescent hepatic stellate cells. Semin Liver Dis. 2001;21(3):311-35.

23. $\mathrm{Xu}$ J, et al. The types of hepatic myofibroblasts contributing to liver fibrosis of different etiologies. Front Pharmacol. 2014;5:167.

24. Tsukada S, Parsons CJ, Rippe RA. Mechanisms of liver fibrosis. Clin Chim Acta. 2006:364(1-2):33-60.

25. Hayashi H, Sakai T. Biological significance of local TGF- $\beta$ activation in liver diseases. Front Physiol. 2012;3:12.

26. Bedossa P, Patel K, Castera L. Histologic and noninvasive estimates of liver fibrosis. Clin Liver Dis. 2015;6(1):5-8.

27. Zheng $\mathrm{H}$, et al. Chlorophyllin modulates gut microbiota and inhibits intestinal inflammation to ameliorate hepatic fibrosis in mice. Front Physiol. 2018;9:1671.

28. Roeb E. Matrix metalloproteinases and liver fibrosis (translational aspects). Matrix Biol. 2018;68-69:463-73.

29. Dewidar B, et al. TGF- $\beta$ in hepatic stellate cell activation and liver fibrogenesis-updated 2019. Cells. 2019;8(11):1419.

30. Johnstone RM. Revisiting the road to the discovery of exosomes. Blood Cells Mol Dis. 2005:34(3):214-9. 
31. Wen D, et al. Mesenchymal stem cell and derived exosome as small RNA carrier and Immunomodulator to improve islet transplantation. J Control Release. 2016;238:166-75.

32. Rong $X$, et al. Human bone marrow mesenchymal stem cells-derived exosomes alleviate liver fibrosis through the Wnt/ $\beta$-catenin pathway. Stem Cell Res Ther. 2019;10(1):019-1204.

33. Isola AL, Chen S. Exosomes: the messengers of health and disease. Curr Neuropharmacol. 2017;15(1):157-65.

34. Dai J, et al. Exosomes: key players in cancer and potential therapeutic strategy. Signal Transduct Target Ther. 2020;5(1):020-00261.

35. Kalluri R, LeBleu VS. The biology, function, and biomedical applications of exosomes. Science. 2020. https://doi.org/10.1126/science.aau6977.

36. LiT, et al. Exosomes derived from human umbilical cord mesenchymal stem cells alleviate liver fibrosis. Stem Cells Dev. 2013;22(6):845-54.

37. Sakaguchi $Y$, et al. Comparison of human stem cells derived from various mesenchymal tissues: superiority of synovium as a cell source. Arthr Rheum. 2005;52(8):2521-9.

38. Bruno S, Chiabotto G, Camussi G. Extracellular vesicles: a therapeutic option for liver fibrosis. Int J Mol Sci. 2020;21(12):4255.

39. Elliott $\mathrm{RO}, \mathrm{He}$ M. Unlocking the power of exosomes for crossing biological barriers in drug delivery. Pharmaceutics. 2021;13(1):122.

40. Das CK, et al. Exosome as a novel shuttle for delivery of therapeutics across biological barriers. Mol Pharm. 2019;16(1):24-40.

41. Kordelas L, et al. MSC-derived exosomes: a novel tool to treat therapyrefractory graft-versus-host disease. Leukemia. 2014;28(4):970-3.

42. Karaoz E, Sun E, Demir CS. Mesenchymal stem cell-derived exosomes do not promote the proliferation of cancer cells in vitro. Int J Physiol Pathophysiol Pharmacol. 2019;11(4):177-89.
43. Rosenberger $L$, et al. Stem cell exosomes inhibit angiogenesis and tumor growth of oral squamous cell carcinoma. Sci Rep. 2019;9(1):018-36855.

44. Romanov YA, et al. Human umbilical cord blood serum: effective substitute of fetal bovine serum for culturing of human multipotent mesenchymal stromal cells. Bull Exp Biol Med. 2017;162(4):528-33.

45. Castellano JM, et al. Human umbilical cord plasma proteins revitalize hippocampal function in aged mice. Nature. 2017;544(7651):488-92.

46. Xu F, et al. TGF- $\beta / S M A D$ pathway and its regulation in hepatic fibrosis. J Histochem Cytochem. 2016;64(3):157-67.

47. $\mathrm{Hu} \mathrm{HH}$, et al. New insights into TGF- $\beta /$ Smad signaling in tissue fibrosis. Chem Biol Interact. 2018;292:76-83.

48. Inagaki Y, Okazaki I. Emerging insights into transforming growth factor beta Smad signal in hepatic fibrogenesis. Gut. 2007;56(2):284-92.

49. Ling MT, et al. Down-regulation of Id-1 expression is associated with TGF beta 1 -induced growth arrest in prostate epithelial cells. Biochim Biophys Acta. 2002;15(3):145-52

50. $\mathrm{MaH}$, et al. TGF- $\beta 1$-induced expression of Id- 1 is associated with tumor progression in gastric cancer. Med Oncol. 2014;31(7):014-0019.

51. Stankic M, et al. TGF- $\beta$-Id1 signaling opposes Twist1 and promotes metastatic colonization via a mesenchymal-to-epithelial transition. Cell Rep. 2013;5(5):1228-42.

\section{Publisher's Note}

Springer Nature remains neutral with regard to jurisdictional claims in published maps and institutional affiliations.
Ready to submit your research? Choose BMC and benefit from:

- fast, convenient online submission

- thorough peer review by experienced researchers in your field

- rapid publication on acceptance

- support for research data, including large and complex data types

- gold Open Access which fosters wider collaboration and increased citations

- maximum visibility for your research: over $100 \mathrm{M}$ website views per year

At BMC, research is always in progress.

Learn more biomedcentral.com/submissions 